\title{
Squareness error calibration of a CMM for quality control of ophthalmic lenses
}

\author{
Diego Rodríguez-Ibáñez • José Alonso • \\ Juan Antonio Quiroga
}

Received: 4 August 2012 / Accepted: 9 January 2013 / Published online: 13 February 2013

(C) The Author(s) 2013. This article is published with open access at Springerlink.com

\begin{abstract}
This paper proposes a calibration procedure for the squareness error of small-sized coordinate measuring machines like those used for ophthalmic lenses quality control. The proposed procedure only requires a spherical lens as a reference surface and it only needs to be measured once making this calibration method faster and cheaper than the existing ones.
\end{abstract}

Keywords CMM - Calibration - Squareness error · Volumetric error · Ophthalmic lenses quality control

\section{Introduction}

Optical methods that measure the wavefront refracted by a lens are well suited for calculating its through power; they are fast and precise. Also, manufacturing quality and optical performance can be usually inferred from the through power. However, when we need the topography of a lens (for ray tracing, for example), the minute curvature errors quickly accumulate as we integrate them over the measured surface. In those cases, coordinate measuring machines (CMMs) are widely used as profilometers to measure lens topography. One of the simplest setups is the column type or Cartesian CMM; it consists of two linear actuators for horizontal positioning and a vertical probe for measuring height. The ones used in ophthalmic lens profilometry have a relatively small working volumes (around $10 \mathrm{~cm}$ each side) and its volumetric error must be below $1 \mu \mathrm{m}$.

D. Rodríguez-Ibáñez $(\bowtie) \cdot$ J. Alonso · J. A. Quiroga

Department of Optics, Universidad Complutense de Madrid, Madrid, Spain

e-mail: diego.rodriguez@ucm.es
While 21 error parameters are needed to calibrate a column-type CMM [1-3], the squareness error can account for more than $50 \%$ of the total error budget [4]. Also, the increasing precision of available linear actuators makes it easy for a laboratory to build its own CMM from offthe-shelf parts. Linear and angular errors can be measured independently for each linear stage and the manufacturer provides their upper bounds in the specifications. This makes the squareness error more relevant as it depends on the precision of the assembly.

Error characterization and compensation for CMMs has been extensively studied, and many methods for squareness error calibration have been already proposed. However, they require the use of previously calibrated artifacts like ball bars or hole plates. Most of these artifacts are expensive or require another more precise CMM to calibrate them [5, 6]. Moreover, existing calibration procedures usually require to measure the reference object in several specific positions along the working volume [4, 7]. This is time consuming and introduces the need of extra equipment to guarantee that the positioning specifications are met.

In this paper, we propose a simpler squareness error calibration method. It uses a spherical reference surface as a calibrating artifact. Accurate glass lenses or calipers can be used as a reference surface; they are cheap and already available and they come pre-calibrated. Also, the reference surface is measured only once and its position and orientation inside the working volume are arbitrary. Not even its radius is needed to be known.

The proposed method, while developed for profilometers, can be used to calibrate any Cartesian CMM as long as the size of the reference surface matches that of the working volume. 


\section{Profilometer model}

Our model of the Cartesian profilometer has two motors for horizontal displacement and a vertical sensor probe. The motors' displacement axes are called $U$ and $V$. The sensor probe axis is called $W$. They define the non-square reference system $O^{\prime}$. The model also includes an ideal Cartesian reference system called $O$, its axes being $X, Y$, and $Z$. For the sake of simplicity, $O$ is set so that $Z$ coincides with $W$ and that $X$ is in the same plane as $U$ and $W$.

Three squareness error parameters are required to fully determine $O^{\prime}$ axes' relative orientations. With this setup, they are $\delta_{U W}$ (squareness error of $U$ in reference to $W$ ), $\delta_{V U}$ (squareness error of $V$ in reference to $U$ ), and $\delta_{V W}$ (squareness error of $V$ in reference to $W$ ). They are represented in Fig. 1 while a cross section of the system can be seen in Fig. 2.

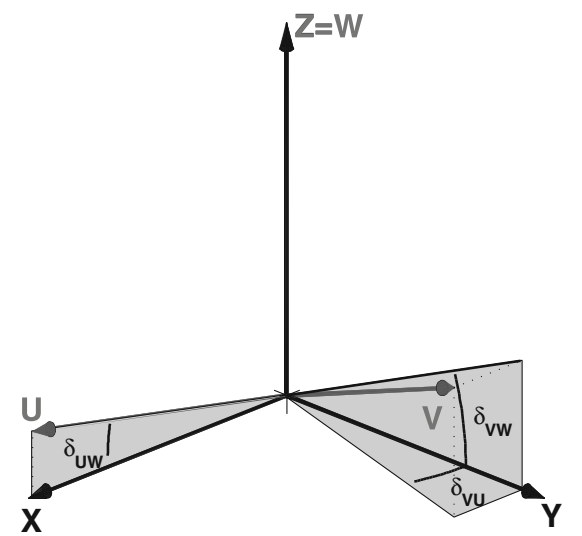

Fig. 1 Relative orientations between $O$ and $O^{\prime}$ axes

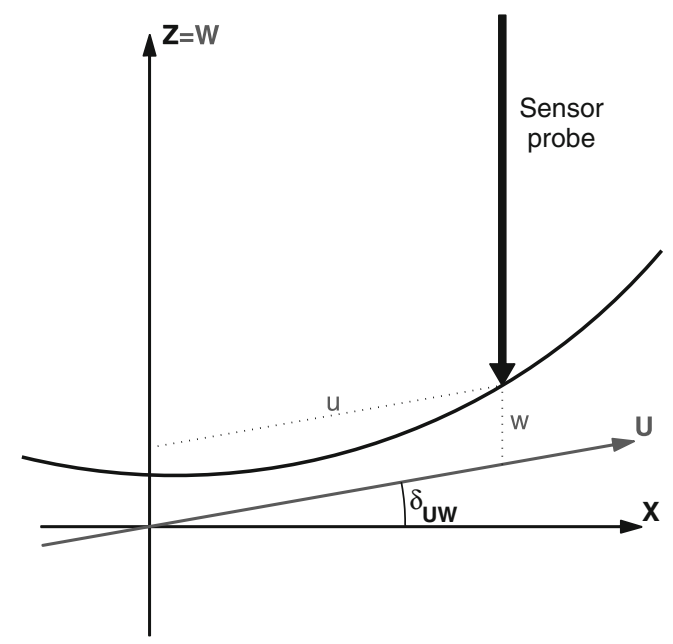

Fig. 2 Cross section along the $X Z$ plane of the modeled profilometer. $X$ and $Z$ are ideal Cartesian axes. $U$ and $W$ are real axes; they are defined by one of the motors and the sensor probe axis, respectively. $w$ is the measured height after a displacement $u$ of the said motor

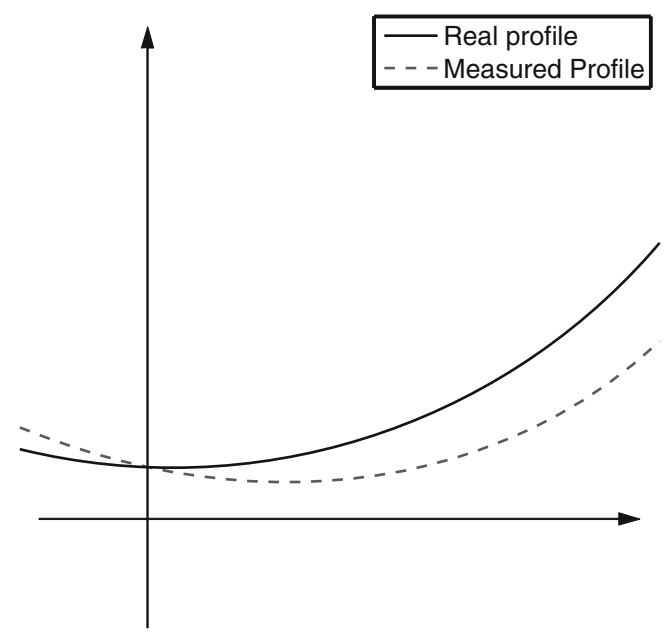

Fig. 3 Comparison between the cross section of a sample sphere (solid) and the height profile expected after measuring it with a non-square profilometer (slashed)

We can translate the coordinates of any given point between both reference systems with

$$
\begin{aligned}
{\left[\begin{array}{l}
x \\
y \\
z
\end{array}\right] } & =T\left[\begin{array}{c}
u \\
v \\
w
\end{array}\right] \\
& =\left[\begin{array}{ccc}
\cos \left(\delta_{U W}\right) & \sin \left(\delta_{V U}\right) \cos \left(\delta_{V W}\right) & 0 \\
0 & \cos \left(\delta_{V U}\right) \cos \left(\delta_{V W}\right) & 0 \\
\sin \left(\delta_{U W}\right) & \sin \left(\delta_{V W}\right) & 1
\end{array}\right]\left[\begin{array}{c}
u \\
v \\
w
\end{array}\right]
\end{aligned}
$$

where $T$ is the transformation matrix, $(u, v, w)$ are the coordinates of a point measured in the $O^{\prime}$ reference system, and $(x, y, z)$ are the coordinates of the same point in $O$.

Table 1 Specifications of the Newport GTS150 linear stages used in the profilometer

\begin{tabular}{ll}
\hline Parameter & Value \\
\hline Travel range & $150 \mathrm{~mm}$ \\
Resolution & $0.05 \mu \mathrm{m}$ \\
Bidirectional repeatability & $0.2 \mu \mathrm{m}$ \\
On axis accuracy & $1 \mu \mathrm{m}$ \\
Straightness, flatness & $1 \mu \mathrm{m}$ \\
\hline
\end{tabular}

Table 2 Calibration results for the first configuration

\begin{tabular}{lll}
\hline Parameter & Mean $\left(10^{-3} \mathrm{rad}\right)$ & $\sigma\left(10^{-3} \mathrm{rad}\right)$ \\
\hline$\delta_{U W}$ & -1.1 & 0.5 \\
$\delta_{V U}$ & -0.05 & 0.05 \\
$\delta_{V W}$ & 29.0 & 0.4 \\
\hline
\end{tabular}






(a)

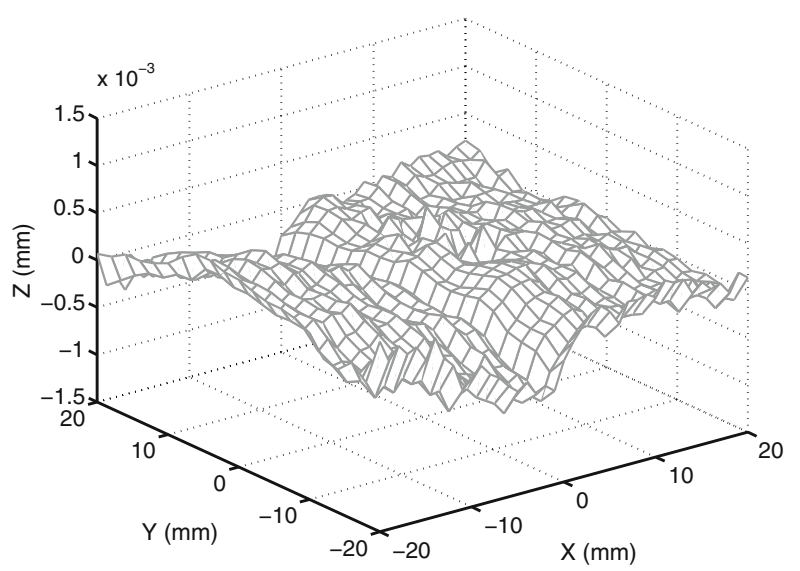

(b)

Fig. 4 Residue comparison before (a) and after (b) the minimization of $\phi$. Please note that the vertical scale is an order of magnitude smaller in $\mathbf{b}$

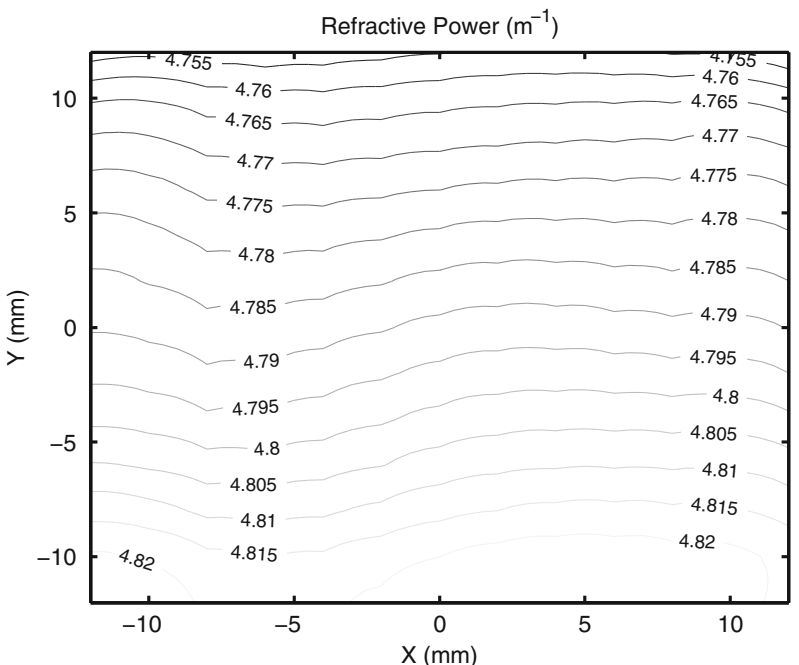

(a)



(c)

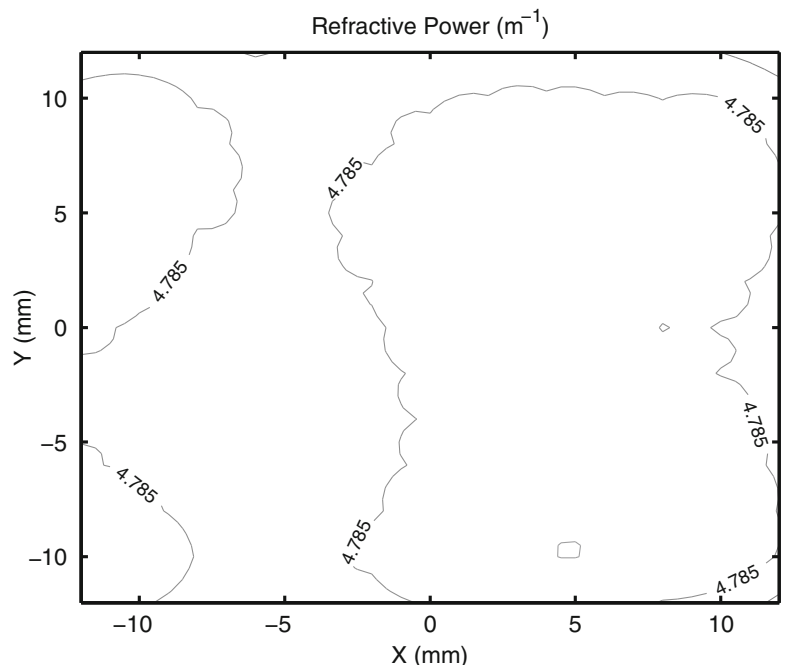

(b)

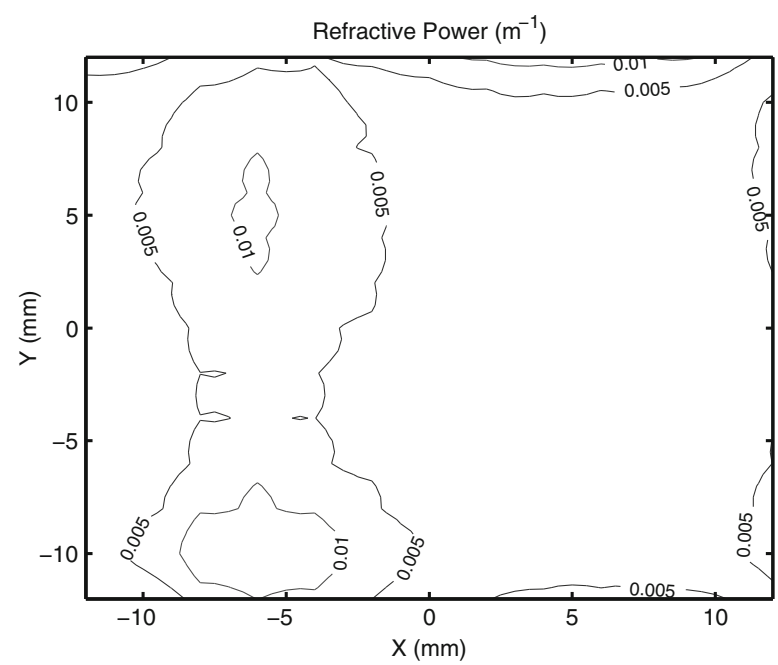

(d)

Fig. 5 Calculated power maps assuming $n=1.5$ for the sample spherical lens and the first profilometer configuration: sphere before (a) and after (b) squareness error correction and cylinder before (c) and after (d) squareness error correction 
Table 3 Calibration results for the second configuration

\begin{tabular}{lll}
\hline Parameter & Mean $\left(10^{-3} \mathrm{rad}\right)$ & $\sigma\left(10^{-3} \mathrm{rad}\right)$ \\
\hline$\delta_{U W}$ & -8.8 & 0.4 \\
$\delta_{V U}$ & 0.00 & 0.05 \\
$\delta_{V W}$ & 19.9 & 0.4 \\
\hline
\end{tabular}

On another hand, the points $\left(x_{S}, y_{S}, z_{S}\right)$ of a generic sphere, $S$, in $O$ must satisfy the following equation:

$\mathcal{S}\left(x_{S}, y_{S}\right)=z_{S}=R\left(1-\sqrt{1-\frac{\left(x_{S}-x_{V}\right)^{2}+\left(y_{S}-y_{V}\right)^{2}}{R^{2}}}\right)+z_{V}$

where $V=\left(x_{V}, y_{V}, z_{V}\right)$ is the vertex of the sphere and $R$ is its radius. Also, note that Eq. 2 is valid for both convex and concave spherical surfaces. $R>0$ defines a convex surface and $R<0$ a concave one.

Substituting Eq. 1 in Eq. 2

$$
\begin{aligned}
\mathcal{S}(x(u, v, w), y(u, v, w)) & =z(u, v, w) \\
& \Rightarrow \mathcal{S}(x(u, v, w), y(u, v, w)) \\
& =u \sin \left(\delta_{U W}\right)+v \sin \left(\delta_{V W}\right)+w
\end{aligned}
$$

and isolating $w$, we get the function

$$
\begin{aligned}
w(u, v)= & \mathcal{S}\left(u \cos \left(\delta_{U W}\right)+v \sin \left(\delta_{V U}\right) \cos \left(\delta_{V W}\right),\right. \\
& \left.v \cos \left(\delta_{V U}\right) \cos \left(\delta_{V W}\right)\right) \\
& -u \sin \left(\delta_{U W}\right)-v \sin \left(\delta_{V W}\right)
\end{aligned}
$$

which models the expected height map obtained when measuring $S$ using a profilometer with given squareness errors $\delta=\left(\delta_{U W}, \delta_{V U}, \delta_{V W}\right)$. Like Eq. 2 , this model is valid for both concave and convex surfaces. Figure 3 shows a cross section of a concave spherical surface along the $X Z$ plane compared with its modeled profile for $\delta_{U W}>0$.

\section{Determining the squareness errors}

Lets assume that we have a series of experimental values $\left\{M_{n}\right\}=\left\{\left(u_{n}, v_{n}, w_{n}\right)\right\}, n=1,2, \ldots, k$ obtained by measuring over a sample sphere. For each possible combination of the parameter set $(R, V, \delta)$, the residue of each point is defined as

$r\left(M_{n} ; R, V, \delta\right)=w_{n}-w\left(u_{n}, v_{n} ; R, V, \delta\right)$.

With this, we build the merit function

$\phi(R, V, \delta)=\sum_{n=1}^{k}\left(r\left(M_{n} ; R, V, \delta\right)\right)^{2}$

This function is a measure of the fitness of the model parameters. The more similar the input parameters are to those of the experimental system, the smaller will the residue be. Our objective becomes to find the combination of $R, V$, and $\delta$ that minimizes $\phi(R, V, \delta)$. This is an optimization problem in a seven-dimensional space. To solve it, we have used an implementation of the simplex algorithm [8]. If the initial values are not adequate, the method may converge to a relative minimum (not the absolute one), but we can assume that the values of $\delta$ are close to 0 and estimate the initial $R$ and $V$ adjusting the data to a non-distorted sphere. The $\delta=\left(\delta_{U W}, \delta_{V U}, \delta_{V W}\right)$ obtained after the minimization contains the squareness error parameters needed for the calibration.

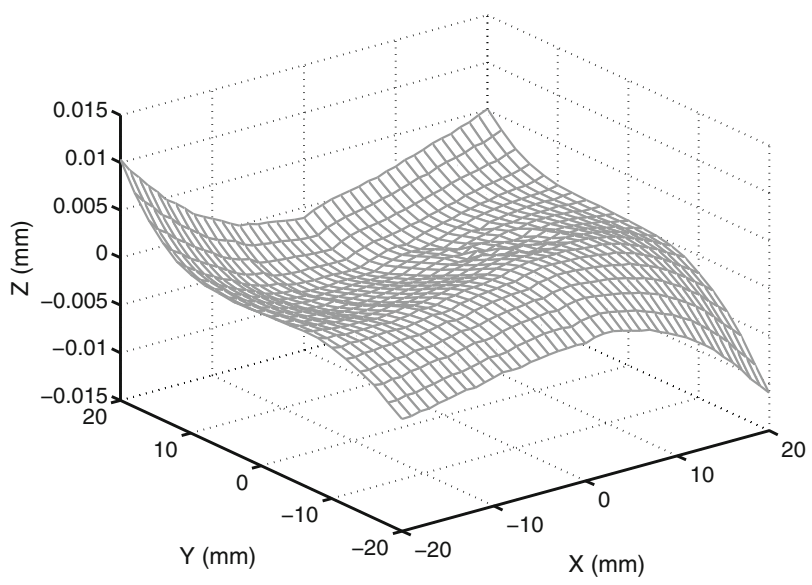

(a)

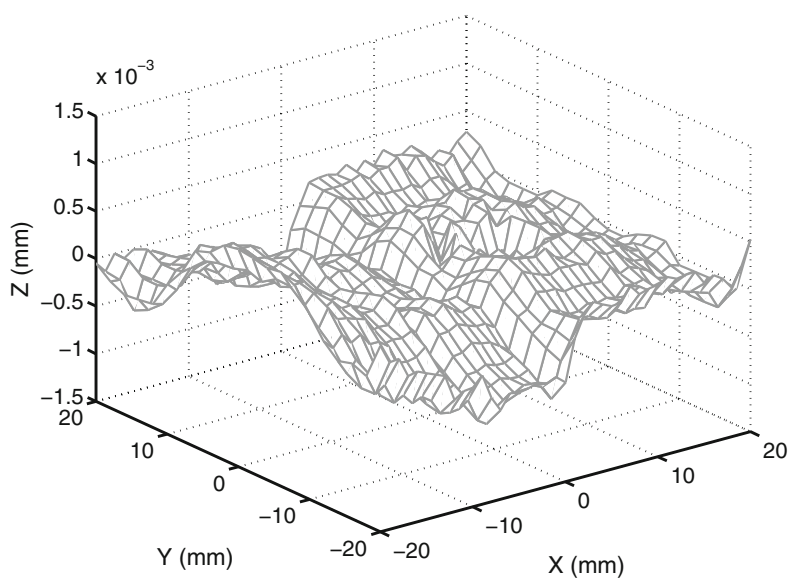

(b)

Fig. 6 Residue comparison before (a) and after (b) the minimization of $\phi$. Please note that the vertical scale is an order of magnitude smaller in $\mathbf{b}$ 




(a)



(c)

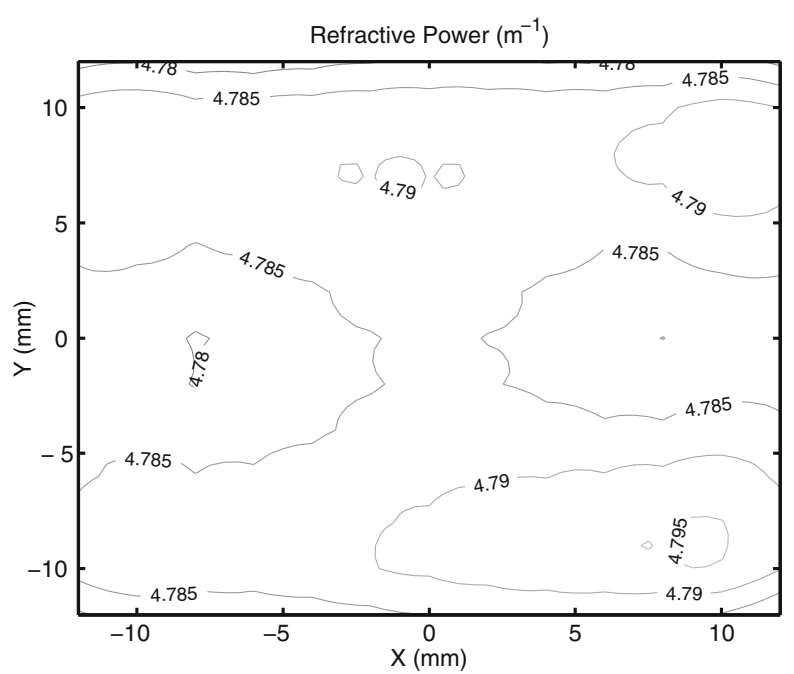

(b)



(d)

Fig. 7 Calculated power maps assuming $n=1.5$ for the sample spherical lens and the second profilometer configuration: sphere before (a) and after (b) squareness error correction and cylinder before (c) and after (d) squareness error correction

\section{Experimental results}

To test the proficiency of the proposed calibration method, we used a custom-made CMM. The actuators are two pre-calibrated Newport GTS150 linear stages. Their specifications are listed in Table 1. They were also pre-assembled with a nominal squareness error $\delta_{V U}=0 \pm 10^{-5} \mathrm{rad}$. The sensor probe is a Heidenhain MT 2587 length gauge with a nominal $\pm 0.2-\mu \mathrm{m}$ accuracy. The reference surface is the convex surface of a precision spherical lens accurate to $\lambda / 8$ within $80 \%$ of its full aperture $(\lambda=633 \mathrm{~nm})$ and its radius is $R=104.5 \pm 0.1 \mathrm{~mm}$. In each calibration run, a grid of $21 \times 41$ points over an area of $20 \times 20 \mathrm{~mm}$ was measured.
Initially, two batches of tests were made with different orientations of the sensor probe axis; each batch consisting of 12 calibration runs with the reference lens in different positions. The values of $\delta$ are first estimated measuring various key dimensions with a caliper applying trigonometry.

Table 4 Calibration results for the third configuration

\begin{tabular}{lll}
\hline Parameter & Mean $\left(10^{-3} \mathrm{rad}\right)$ & $\sigma\left(10^{-3} \mathrm{rad}\right)$ \\
\hline$\delta_{U W}$ & 6 & 1 \\
$\delta_{V U}$ & 7.4 & 0.2 \\
$\delta_{V W}$ & 21.9 & 0.4 \\
\hline
\end{tabular}




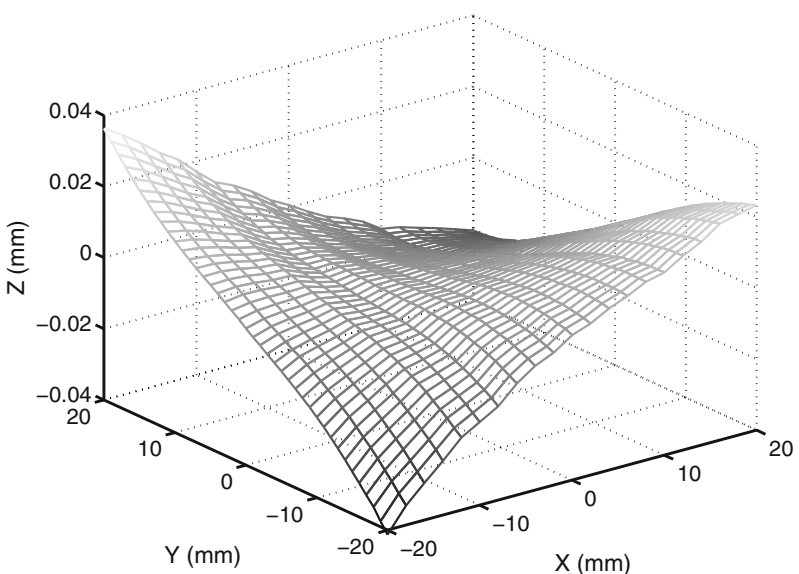

(a)

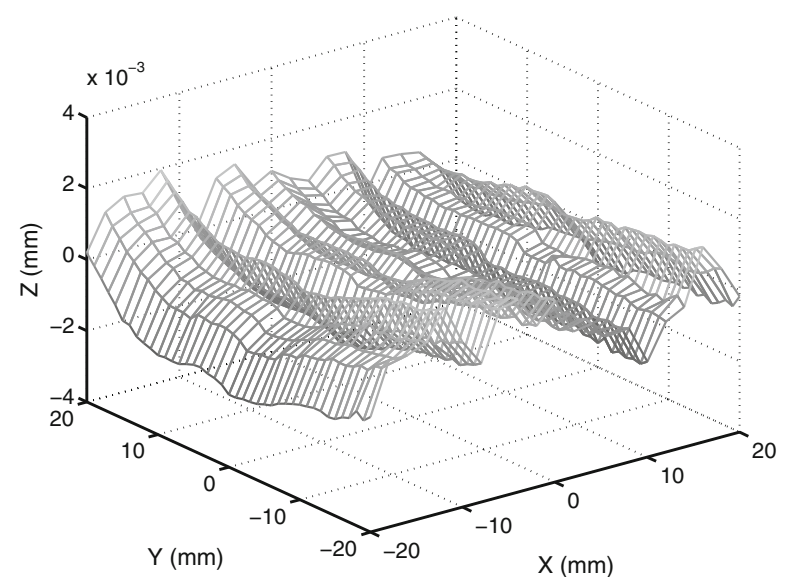

(b)

Fig. 8 Residue comparison before (a) and after (b) the minimization of $\phi$. Please note that the vertical scale is an order of magnitude smaller in $\mathbf{b}$

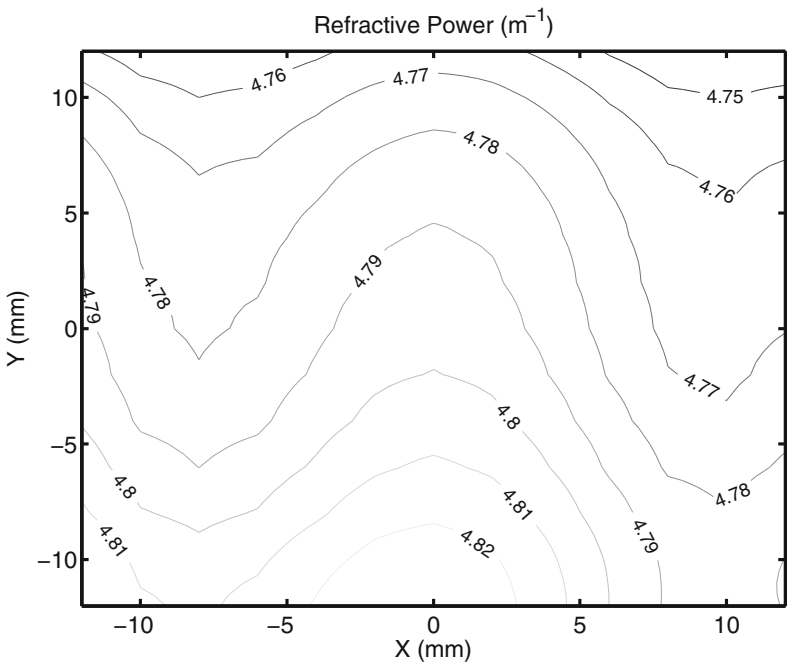

(a)

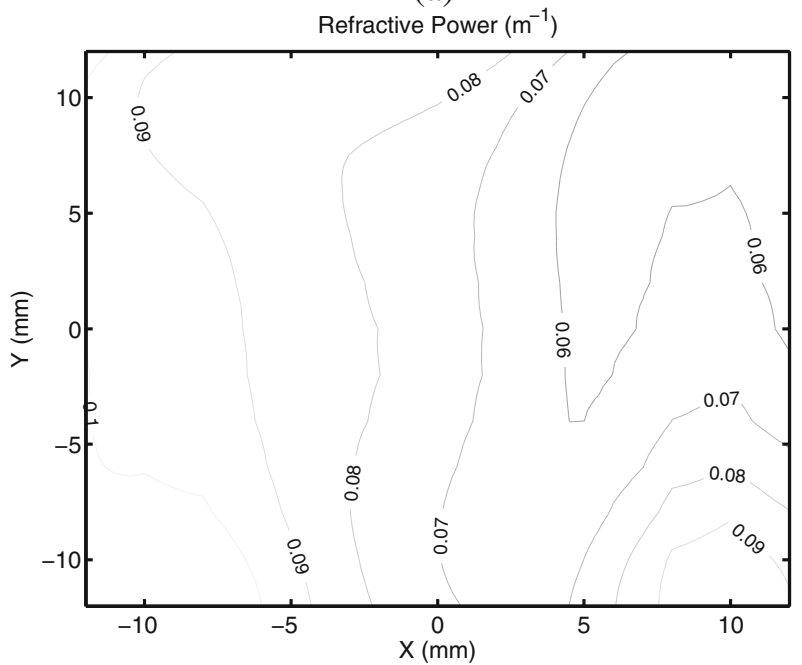

(c)

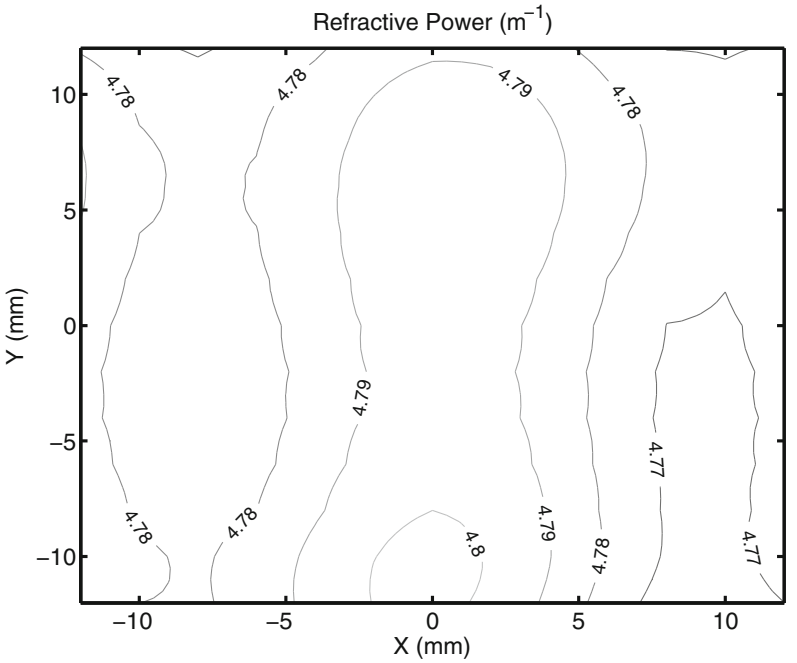

(b)

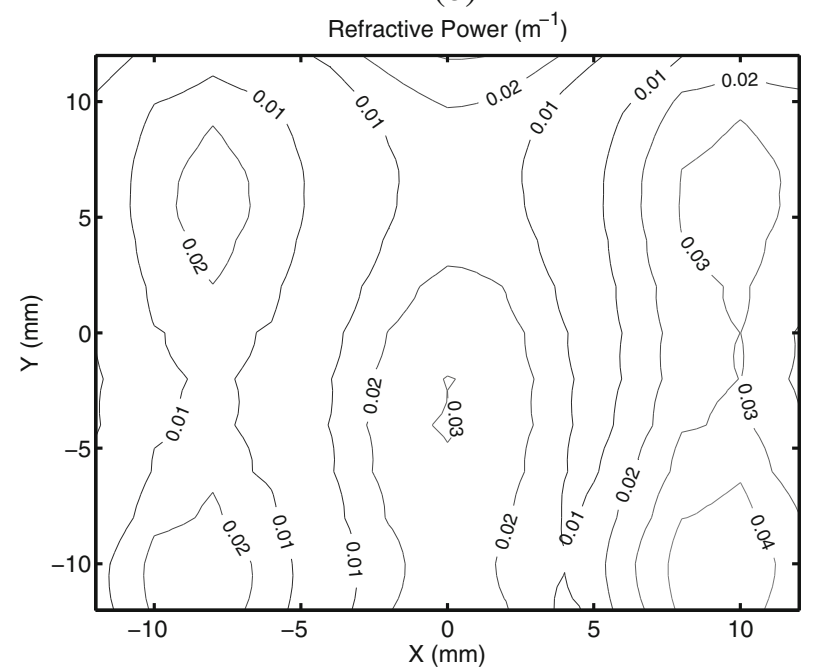

(d)

Fig. 9 Calculated power maps assuming $n=1.5$ for the sample spherical lens and the third profilometer configuration: sphere before (a) and after (b) squareness error correction and cylinder before (c) and after (d) squareness error correction 
Then, $\delta$ is calculated with the proposed method. It is also worth noting that the average time elapsed during the residue minimization of each calibration run is $0.4 \mathrm{~s}$.

For the first configuration, the estimated error parameters are $\delta_{U W}=0.000 \pm 0.004 \mathrm{rad}, \delta_{V U}=0 \pm 10^{-5} \mathrm{rad}$, and $\delta_{V W}=0.032 \pm 0.004 \mathrm{rad}$.

The error parameters obtained from the calibration process can be seen in Table 2, while Fig. 4 shows the residues of a sample calibration run before and after minimizing $\phi$ and Fig. 5 shows the refractive power maps calculated from the same data.

For the second configuration, the estimated error parameters are $\delta_{U W}=-0.010 \pm 0.004 \mathrm{rad}, \delta_{V U}=0 \pm 10^{-5} \mathrm{rad}$, and $\delta=0.019 \pm 0.004 \mathrm{rad}$.

The error parameters obtained from the calibration process can be seen in Table 3 while Fig. 6 shows the residues of a sample calibration run before and after minimizing $\phi$ and Fig. 7 shows the refractive power maps calculated from the same data.

A third batch of tests was needed to test the proficiency of the method when $\delta_{V U} \neq 0$. We had to change the setup used previously because the angle between both Newport GTS150 linear stages was locked. We substituted one of those stages with a manual linear stage mounted on a goniometer. The manual stage has a micrometric screw with a precision of $10 \mu \mathrm{m}$. Its displacement axis then defines $U$, the displacement axis of the remaining motor stage defines $V$, and we can rotate $U$ inside the horizontal plane using the goniometer.

For our purposes, this setup is essentially the same with the two first cases that we have used, the main difference being that the precision along the $U$ axis is $10 \mu \mathrm{m}$ instead of $1 \mu \mathrm{m}$. As in the previous test cases, we made 12 calibration runs with the reference lens in different positions.

The estimated error parameters from geometrical measures are $\delta_{U W}=0.008 \pm 0.004 \mathrm{rad}, \delta_{V U}=0.009 \pm$ $0.004 \mathrm{rad}$, and $\delta_{V W}=0.019 \pm 0.004 \mathrm{rad}$.

The error parameters obtained from the calibration process can be seen in Table 4, while Fig. 8 shows the residues of a sample calibration run before and after minimizing $\phi$ and Fig. 9 shows the refractive power maps calculated from the same data.

The ridges in Fig. $8 \mathrm{~b}$ appear because of the lesser positioning precision along the $U$ axis. However, the residue structure is more sensible to $\delta_{V U}$ than it is to $\delta_{U W}$ or $\delta_{V W}$, so the consistency of the calculated $\delta_{V U}$ is greater than that of $\delta_{U W}$ or $\delta_{V W}$ in the previous cases despite the loss in precision.

\section{Conclusions}

The squareness errors in the profilometer produce very significant volumetric errors in the measured surface. These measurement errors translate into perceptible errors in the calculated power maps.

The proposed method successfully corrects the squareness errors and any deviations derived from them up to the required specifications. It introduces the use of reference surfaces that are already common and, therefore, relatively cheap. Only one measuring round is needed and there is no precise positioning required. This reduces the workload of the operators and eliminates the need of extra measuring instruments. Also, the processing time used during the minimization of the merit function is negligible and the results are consistent. All these features make this calibration process time and cost-effective.

Acknowledgments This work was supported by the Spanish Ministry of Science and Technology under grant DPI2009-09023.

Open Access This article is distributed under the terms of the Creative Commons Attribution License which permits any use, distribution, and reproduction in any medium, provided the original author(s) and the source are credited.

\section{References}

1. Barakat NA, Elbestawi MA, Spence AD (2000) Kinematic and geometric error compensation of a coordinate measuring machine. Int J Mach Tools Manuf 40(6):833-850

2. Okafor AC, Ertekin YM (2000) Derivation of machine tool error models and error compensation procedure for three axes vertical machining center using rigid body kinematics. Int $\mathrm{J}$ Mach Tools Manuf 40(8):1199-1213

3. Ramesh R, Mannan MA, Poo AN (2000) Error compensation in machine tools-a review: part I: geometric, cutting-force induced and fixture-dependent errors. Int J Mach Tools Manuf 40(9): $1235-1256$

4. Wand BT, Bressler VE, Ruland R Squareness error mapping of a coordinate measuring machine using a two-dimensional reference object, SLAC Publication, SLAC-PUB-5836

5. Lee ES, Burdekin M (2001) A hole-plate artifact design for the volumetric error calibration of CMM. Int J Adv Manuf Technol 17:508-515. doi:10.1007/s001700170151

6. Liebrich T, Bringmann B, Knapp W (2009) Calibration of a 3D-ball plate. Precision Engineering 33(1):1-6

7. Kruth JP, Zhou L, Van den Bergh C, Vanherck P (2003) A method for squareness error verification on a coordinate measuring machine. Int J Adv Manuf Technol 21:874-878. doi:10.1007/ s00170-002-1408-X

8. Lagarias JC, Reeds JA, Wright MH, Wright PE (1998) Convergence properties of the nelder mead simplex method in low dimensions. SIAM J Optim 9(1):112-147 